\title{
Preliminary studies on students' understanding of electricity and magnetism for the development of a model based diagnostic instrument
}

\author{
Rasil Warnakulasooriya and Lei Bao \\ Dept. of Physics, The Ohio State University, $174 \mathrm{~W} .18^{\text {th }}$ Avenue, Columbus, OH 43210
}

Learning is context dependent. Hence, the development of diagnostic tools should follow from extensive qualitative research that reveals the subtle relations between contexts and the development of students' knowledge. In this paper, we present initial results within the framework of developing a model based diagnostic instrument on electricity and magnetism.

\section{Introduction}

Electricity and magnetism (E\&M) poses many challenges in both learning and instruction. It has many inherent conceptual difficulties (e.g., the idea of fields and potentials) and the level of technical expertise needed to solve problems effectively and successfully is demanding for most students. Many studies have been carried out from both the learning and instructional perspectives.

Among these studies, students' understanding of electric circuits is one of the areas that has been researched extensively. McDermott and Shaffer ${ }^{1}$ investigated students' understanding of circuits as a guide in curriculum development while Bagno and Eylon ${ }^{2}$, Ferguson-Hessler and de Jong ${ }^{3}$ concentrated on the students' knowledge structure and its construction in the context of E\&M.

Other areas have also been explored. For example, Törnkvist, Pettersson and Tranströmer ${ }^{4}$ had studied students' understanding of the electric field from a representational point of view. The study concludes that students tend to attach much more reality to field lines than is desired.

Research-based assessment instruments have also been developed. A good example is the Conceptual Survey of Electricity and Magnetism $(\mathrm{CSEM})^{5}$, which provides a powerful tool to probe a wide range of issues of student understanding of E\&M.
In our research on E\&M, we systematically investigate the context dependency of students' learning and how context issues affect students' understanding. This is the initial step towards developing an assessment tool for E\&M based on the method of Model Analysis ${ }^{6}$.

In Model Analysis, the collection of students' mental models forms the model space. The identification of the model space and the development of a diagnostic instrument based on it may help instructional techniques to address these particular models effectively. We believe that such a scheme will make students select the correct models with a higher probability at the end of instruction.

\section{Framework}

To develop diagnostic questions based on Model Analysis, extensive qualitative research has to be done to identify the common student models. We try to identify possible models through student responses to the questions based on physical concepts. Let us call these concepts as concept topics. Therefore, the identification of concept topics forms an essential part of our initial work.

The selection of concept topics is primarily based on the fundamental principles of physics. Possible student models and the relevant context issues are identified through informal discussions, 
student responses in recitations, homework problems and exams. Topics connecting one domain to another that help students see physics in a unified manner were emphasized. Once a topic is determined, we make questions that would help us identify the possible mental models and the study is conducted with a single concept topic at a time. The questions may be multiple choice and/or open-ended and are implemented through web-based surveys and interviews.

Associated with a single concept topic are the context issues that affect the activation and application of mental models. The context dependency of students' initial models in general, is related to their experience. By studying the real life examples that students use in their reasoning, we can extract valuable information on the context issues of the student models. Therefore, one aspect to our study is to survey the meanings and explanations that students attach to everyday physical phenomena involving E\&M. It is important to recognize how the level of abstractness of a question would trigger different responses. This in turn helps us select, fine-tune and phrase questions in the best ways possible.

In our research, we also study how students 'move' across two conceptual domains, and how the different physical contexts that were originally used to introduce the physics concepts may affect students' knowledge across different domains. An example is the inclusion of Newtonian mechanics as a 'subset' in the study of $E \& M^{7}$. Specifically, we investigate whether students tend to keep the classical mechanical and electromagnetic domains separate or if they transfer knowledge from one to the other, then to what extent they carry ideas across and what inherent difficulties (especially with respect to contexts) are associated with such transitions. The importance of this study lies in the fact that a particular response can then be properly attributed to its specific domain or as a transitional difficulty between the domains, which will facilitate the instructor to better understand the possible causes of student difficulties. Moreover, it helps us build better (clean) questions for the diagnosis purposes.

We now turn to some concept topic examples. Given the limited space, we summarize our observations. The data that we had gathered thus far results from an algebra-based E\&M course offered at The Ohio State University.

\section{Concept Topics}

- The meaning of the word "static" in different contexts: We investigate the meaning students attach to 'static' in day-today experience and in the context of static electricity as introduced in textbooks in the discussions of Coulomb's law. In the former context, answers were consistent with an extra charge build-up (static shocks). In the latter context, answers ranged from 'stationary' to 'not continuous'. The 'not continuous' idea was stated in the context of static on the television or the radio, whence the receiving signal is not continuous (smooth). Some drew analogies to 'static friction'. The experience of static cling of clothes was taken to give meaning to static electricity as attraction/repulsion.

- Behavior of charges in $\mathbf{E}$ and $\mathbf{B}$ fields: The results suggest that most of the students carry their knowledge of $\mathbf{E}$ fields into the realm of $\mathbf{B}$ fields directly. Many students answered that a charge placed at rest in a $\mathbf{B}$ field would move in the direction of the field. Most students tend to attach meaning to the field arrows as giving a 'kick' to the charges. Furthermore, many students do not seem to realize that a magnetic field is created only by the moving charges. This was revealed through questions that asked students to identify the fields produced by charges under relative motion. However, further study is required to see whether the difficulties are due to the relative motion 
itself or whether it is an inherent difficulty in understanding electromagnetics alone.

- Electromagnetic induction and Lenz's law: Many fail to identify that it is the changing flux through a coil that leads to an induced voltage and could not attribute it to any work-energy relationship. This suggests difficulties associated in moving between knowledge domains and seeing physics in a unified way.

- Charge conservation: The question given relates to an example from a particle physics experiment. The experiment involves a collision between a positive and a negative charge. We asked students to state the net charge of the particles being created from the collision. Almost all stated the correct answer, without explicitly stating the principle of charge conservation. It seems natural for students to consider algebraic addition of positive and negative numbers (charges) without relating to a physical principle.

\section{A Detailed Example}

In order to give the reader a clearer sense of the way our work is carried out, let us turn to a discussion of a concept topic in detail - the stability of matter on the basis of the Coulomb forces.

On the basis of the Coulomb force alone we would expect the positive nuclei and the electrons to collide. However, from a student perspective the topic involves many issues that can affect their responses. For example, students have studied mechanics and chemistry. Most are familiar with the solar system model of the atom. Some may even be aware of the quantum principles at work. Within E\&M alone, we wanted to see how students attach meaning to electric fields and the ideas of attraction (between nuclei and electrons) and repulsion (among electrons). Thus we expected the concept topic to shed light on student models, their reasoning, domain shifts and the interplay between abstractness versus their experience. The question we posed was: You have learned that charges of same type repel and that charges of different types attract. All objects in the world are made up of atoms, which consist of positive nuclei and negative electrons. If positive and negative charges attract each other, will the nuclei and electrons collide and land on each other?

All students stated that they would not collide. However, after stating so, some would speculate that if they do collide then it would result in a nuclear reaction. The $\mathbf{E}$ field of the nuclei keeping the electrons away from it seems to be a common belief. This suggests a lack of understanding of the principles but a possible model - a 'shield' to prevent collision. Other ideas include electrons repelling each other and driving them far apart from the nucleus and arguing that the atom as a whole is neutral.

Classical mechanics was also used, stating that centrifugal force keeps the electrons away. Analogies were also given: Electrons orbiting the nucleus are similar to the moon orbiting the earth. An interesting revelation though is that in a similar analogy, one student states that:

"Electrons maintain an orbit just as the gravitational pull of the sun does not pull the earth towards it."

It seems that the student fails to identify the importance of the central force in maintaining the orbital (tangential) motion. The weakening of the Coulomb force due to the presence of neutrons in the nucleus was also stated. Quantum ideas were explicitly stated only once.

Given the question (context) considered, we see how the students respond, sometimes moving across domains (solar system analogies and classical mechanics) and taking ideas from the same concept settings such as the $\mathbf{E}$ fields. We note that this type of study yields a collection of ideas that students hold. Our next step is to identify the significant (common) models from this pool in such a way that a given diagnostic 
question could reflect these models in the best way possible. It should be stated that questions, which failed to provide any anticipated results also form an integral part of the study. They help us identify further context dependency issues and how possible restructuring of the questions may be carried out.

\section{Goals Ahead}

The future stages of this research will try to identify and investigate more concept topics such as potential energy, potential difference, "magnetic charges", superposition, etc. The identification of the common models through the concept topic studies will involve a major part of our research. Questions will then be developed taking into account these models and student representations. The questions developed will be fine-tuned based on both qualitative and quantitative research to yield a 'faithful' representation of the students' knowledge states. This may involve several iterations, going through the steps mentioned above. This will lead us to a final set of diagnostic questions, which will be implemented, tested and compared with the existing diagnostic tests.

\section{Acknowledgements}

We thank Dr. Klaus Honscheid for helping us conduct the surveys and the Physics Education Research group at The Ohio State University for their helpful comments. This work is supported in part by NSF grant \#REC-0087788.
${ }^{1}$ L. C. McDermott and P. S. Shaffer, "Research as a guide for curriculum development: An example from introductory electricity. Part I: Investigation of student understanding," Am. J. Phys. 60, 994-1003 (1992); erratum, ibid. 61, 81 (1993).

${ }^{2}$ Esther Bagno and Bat-Sheva Eylon, "From problem solving to a knowledge structure: An example from the domain of electromagnetism,” Am. J. Phys. 65 (1997).

${ }^{3}$ M. G. M. Ferguson-Hessler and T. de Jong, "On the quality of knowledge in the field of electricity and magnetism," Am. J. Phys. 55, 492-497 (1997).

${ }^{4}$ S. Törnkvist, K. -A. Pettersson and G. Tranströmer, "Confusion by representation: On students' comprehension of the electric field concept," Am. J. Phys. 61, 335-338 (1997).

${ }^{5}$ D. P. Maloney, T. L. O'Kuma, C. J. Hieggelke and A. Van Heuvelen, "Surveying students' conceptual knowledge of electricity and magnetism," Phys. Educ. Res., Am. J. Phys. Suppl., 69 (7), S12-S23, (2001).

${ }^{6}$ L. Bao and E. F. Redish, "Model Analysis: Assessing the dynamics of student learning." Submitted to Cognition and Instruction (2001).

${ }^{7}$ I. Galili, "Mechanics background influences students' conceptions in electromagnetism," Int. J. Sci. Educ. 17:3, 371-387 (1995). 